G.D. DIKSHIT

KODDAI MATH. SEM. REP.

27 (1976), 84-93

\title{
ON ABSOLUTE RIESZ SUMMABILITY FACTORS OF FOURIFR SERIES
}

\author{
BY G. D. DiKshit
}

\section{Definitions and Notations.} write

Let $\Sigma u_{n}$ be an infinite series and let $0=\lambda_{0}<\lambda_{1}<\cdots<\lambda_{n} \rightarrow \infty$. For $k \geqq 0$, we

$$
R^{k}\left(\lambda_{m}\right)=\sum_{n=1}^{m-1}\left(1-\frac{\lambda_{n}}{\lambda_{m}}\right)^{k} u_{n}
$$

The series $\sum u_{n}$ is said to be absolutely summable by Riesz discrete method $\left(R^{*}, \lambda_{n}, k\right)$, or summable $\left|R^{*}, \lambda_{n}, k\right|$, if $\left\{R^{k}\left(\lambda_{n}\right)\right\}$ is of bounded variation. When the 'order' of summation $k=1$, the method of summation is equivalent to the usually known Riesz method $\left|R, \lambda_{n}, 1\right|$. In this specialcase the method is also sometimes known as the method $\left|R, \mu_{n}\right|$, or the method $\left|\bar{N}, \mu_{n}\right|$, where $\left\{\mu_{n}\right\}=$ $\left\{\lambda_{n}-\lambda_{n-1}\right\}$. In this paper we are concerned with this special case and we will denote the method of summation by $\left|R, \lambda_{n}, 1\right|$. Thus the series $\Sigma u_{n}$ is summable $\left|R, \lambda_{n}, 1\right|$ if

$$
\sum_{1}^{\infty}\left|\Delta R^{1}\left(\lambda_{m}\right)\right|=\sum_{m=1}^{\infty}\left(\frac{1}{\lambda_{m}}-\frac{1}{\lambda_{m+1}}\right)\left|\sum_{n=0}^{m} \lambda_{n} u_{n}\right|<\infty .
$$

When $\left\{\lambda_{n}\right\}=\{n\}$, the method is the same as the Cesàro method $|C, 1|$, and when $\left\{\lambda_{n}\right\}=\{\log n\}$ the method is called the logarithmic method.

Let $f(t)$ be a Lebesgue integrable $2 \pi$-periodic function and let the Fourier series of $f(t)$ be given by

We set

$$
f(t) \sim \frac{a_{0}}{2}+\sum_{1}^{\infty}\left(a_{n} \cos n t+b_{n} \sin n t\right)=\sum_{0}^{\infty} A_{n}(t) .
$$

$$
\phi(t)=\frac{1}{2}\{f(x+t)+f(x-t)-2 f(x)\},
$$

and throughout the paper we write to denote

$$
\begin{gathered}
\Phi(t)=\int_{0}^{t}|\phi(u)| d u, \\
p_{n}=\int_{1 / n}^{\pi} \frac{|\phi(u)| d u}{u},
\end{gathered}
$$

Received Feb. 15, 1974. 


$$
\begin{aligned}
D_{n}(t) & =\frac{1}{2}+\cos t+\cos 2 t+\cdots+\cos n t, \\
E_{n}(t) & =\sum_{0}^{n} \lambda_{\nu} \cos \nu t, \\
F_{n}(t) & =\sum_{0}^{n} \varepsilon_{\nu} \lambda_{\nu} \cos \nu t, \\
S_{n}(t) & =\sum_{0}^{n} A_{\nu}(t), \\
h_{n} & =\left\{\begin{array}{lll}
(\log n)^{\beta+\frac{1}{2}}, & \text { when } & \beta>-\frac{1}{2} \\
(\log \log n)^{\frac{1}{2}}, & \text { when } & \beta=-\frac{1}{2} \\
1, & \text { when } & \beta<-\frac{1}{2}
\end{array}\right. \\
k_{n} & =\left\{\begin{array}{lll}
(\log n)^{\beta+1}, & \text { when } & \beta>-1 \\
\log \log n, & \text { when } \quad \beta=-1 \\
1, & \text { when } \quad \beta<-1 .
\end{array}\right.
\end{aligned}
$$

$K, K_{1} \cdots$ denote absolute constants, not necessarily the same, at different occurrences.

\section{Theorems.}

The object of this paper is to establish some general results concerning absolute summability factors for Fourier series at a point. Actually we prove the following theorems:

THEOREM 1. Let $\phi(t)$ be a positive function and let

$$
\Phi(t)=O(t \psi(1 / t)), \quad \text { as } t \rightarrow 0,
$$

and

$$
\phi_{n}=\psi(n)
$$

If $\left\{\varepsilon_{n}\right\}$ is such that

and

(a) $\sum \frac{\mu_{n+1}}{\lambda_{n+1}}\left|\varepsilon_{n}\right| \psi_{n}<\infty, \sum_{1}^{\infty}\left|\Delta \varepsilon_{\nu}\right| \lambda_{\nu} \sum_{\nu+1}^{\infty} \phi_{n} \Delta\left(\frac{1}{\lambda_{n}}\right)<\infty$,

(b) $\sum_{1}^{\infty}\left|\Delta \varepsilon_{\nu}\right| \lambda_{\nu} \sum_{\nu+1}^{\infty} p_{n} \Delta\left(\frac{1}{\lambda_{n}}\right)<\infty$

(c) $\sum_{1}^{\infty} \Delta\left(\frac{1}{\lambda_{n}}\right)\left|\varepsilon_{n} \sum_{1}^{n} \mu_{\nu} \int_{1 / n}^{\pi} \phi(t) D_{\nu-1}(t) d t\right|<\infty$

then $\sum \varepsilon_{n} A_{n}(t)$, at $t=x$, is summable $\left|R, \lambda_{n}, 1\right|$, if, and only if $\sum \frac{\mu_{n+1}}{\lambda_{n+1}}\left|\varepsilon_{n}\right| \mid S_{n}(x)-$ $f(x) \mid<\infty$. 
Theorem 2. Let $\beta$ be a real number, and

$$
\Phi(t)=O\left(t\left(\log \frac{2 \pi}{t}\right)^{\beta}\right), \quad \text { as } t \rightarrow 0 .
$$

If $\left\{\varepsilon_{n}\right\}$ is such that

and
(i) $\Sigma \frac{\left|\varepsilon_{n}\right|(\log n)^{\beta}}{n}<\infty$

(ii) $\sum k_{n}\left|\Delta \varepsilon_{n}\right|<\infty$

then $\sum \varepsilon_{n} A_{n}(t)$, at $t=x$, is summable $|C, 1|$, if, and only if

$$
\Sigma \frac{\left|\varepsilon_{n}\right|}{n}\left|S_{n}(x)-f(x)\right|<\infty \text {. }
$$

THEOREM 3. Let $\beta$ be a real number, and

If $\left\{\varepsilon_{n}\right\}$ is such that

$$
\Phi(t)=O\left(t\left(\log \frac{2 \pi}{t}\right)^{\beta}\right), \quad \text { as } t \rightarrow 0 .
$$

and

(i) $\Sigma \frac{\left|\varepsilon_{n}\right|}{n(\log n)^{1-\beta}}<\infty$

(ii) $\sum k_{n}\left|\Delta \varepsilon_{n}\right|<\infty$,

then $\sum \varepsilon_{n} A_{n}(t)$, at $t=x$, is summable $|R, \log n, 1|$, if, and only if

$$
\sum \frac{\varepsilon_{n}}{n \log n}\left|S_{n}(x)-f(x)\right|<\infty \text {. }
$$

THEOREM 4. Let $\beta$ be any real number and

$$
\Phi(t)=O\left(t\left(\log \frac{2 \pi}{t}\right)^{\beta}\right), \quad \text { as } t \rightarrow 0,
$$

The series $\sum \varepsilon_{n} A_{n}(t)$, at $t=x$, is

(I) summable $|C, 1|$ if $\left\{\varepsilon_{n}\right\}$ is such that

(i) $\left\{\varepsilon_{n} h_{n}\right\} \in B$, (ii) $\sum \frac{\left|\varepsilon_{n}\right| h_{n}}{n}<\infty$ and (iii) $\Sigma k_{n}\left|\Delta \varepsilon_{n}\right|<\infty$;

(II) summable $|R, \log n, 1|$, if $\left\{\varepsilon_{n}\right\}$ is such that

(i) $\left\{\frac{\varepsilon_{n} h_{n}}{\log n}\right\} \in B$, (ii) $\sum \frac{\left|\varepsilon_{n}\right| h_{n}}{n \log n}<\infty$ and (iii) $\Sigma k_{n}\left|\Delta \varepsilon_{n}\right|<\infty$.

\section{Remarks.}

1. For some of the existing results in this direction one is referred to Cheng [2], Prasad and Bhatt [9], Matsumoto [7], Pati [8], Liu [6], Hsiang [5] and S.L. Wang [10]. The special case $\beta=0$ of Theorem 2 was discussed by Pati [8]. Even for the special case, our theorem furnishes more general results than one due to Pati [8].

A theorem on the lines of Theorem 4 for general sequences $\left\{\lambda_{n}\right\}$ can be deduced from Theorem 1 and it can easily be verified that for corresponding 
logarithmico-exponential sequences dealt with in a known theorem due to Matsumoto [7, Theorem 2] here one gets more general results.

2. It has been proved elsehwere [3, Lemma 4] that a sufficient condition for summability $\left|R, \lambda_{n}, 1\right|$ of a series $\sum u_{n}$ is the convergence of $\sum \frac{\mu_{n}}{\lambda_{n}}\left|s_{n}\right|$, $s_{n}=\sum_{0}^{n} u_{k}$. From Theorem 3 we deduce (cf. Corollary to Theorem 3) that when $\lambda_{n}=\log n$, for the summability of the Fourier series of $f(x)$ at the point $t=x$, given by $\Phi(t)=O\left(t\left(\log \frac{1}{t}\right)^{-\eta}\right)$, as $t \rightarrow 0, \eta>0$, this condition is also necessary.

3. The case $\beta \geqq 0$ of Theorem 4 (I), on summability $|C, 1|$, has been studied by Prasad and Bhatt [9]. The summability factors studied in the present paper are sharper than those known before. The case $-1 \leqq \beta \leqq 0$ of the same part has been discussed by Liu [6] whose results are again generalised here. Hsiang [5] rediscovered the case $\beta=-1$ and his result seems to be included in that of Liu [6].

\section{Lemmas.}

We shall need the following results towards the proof of our theorems.

LEMMA 1.

$$
\begin{aligned}
D_{n}(t) & =\frac{\sin \left(n+\frac{1}{2}\right) t}{2 \sin \frac{1}{2} t} \\
& =\left\{\begin{array}{l}
O(n), \\
O\left(\frac{1}{t}\right), t \in\left[\frac{1}{n}, \pi\right] ;
\end{array}\right.
\end{aligned}
$$

(ii) $E_{n}(t)=\lambda_{n} D_{n}(t)-\sum_{1}^{n} \mu_{\nu} D_{\nu-1}(t)$

$$
=\left\{\begin{array}{l}
O\left(n \lambda_{n}\right) \\
O\left(t^{-1} \lambda_{n}\right), t \in\left[\frac{1}{n}, \pi\right] ;
\end{array}\right.
$$

(iii) $F_{n}(t)=\varepsilon_{n} \lambda_{n} D_{n}(t)-\varepsilon_{n} \sum_{1}^{n} \mu_{\nu} D_{\nu-1}(t)+\sum_{0}^{n-1} E_{\nu}(t)\left(\Delta \varepsilon_{\nu}\right)$

$$
=O\left(n \lambda_{n}\left|\varepsilon_{n}\right|\right)+O\left(\sum_{0}^{n-1} \nu \lambda_{\nu}\left|\Delta \varepsilon_{\nu}\right|\right) \text {. }
$$

These results are rather obvious.

LEMma 2. Methods of summation $|R, \log n, 1|$ and $\left|R,(\log n)^{r}, 1\right|, r>0$, are equivalent.

This result can be deduced as a very special case of well-known results on "Second Theorems of consistency for absolute Riesz summability" (cf. [1]). 
LEMMA 3. If $\Phi(t)=O\left(t\left(\log \frac{2 \pi}{t}\right)^{\beta}\right)$, as $t \rightarrow 0$, then

$$
\sum_{0}^{n}\left|S_{\nu}(x)-f(x)\right|=O\left(n h_{n}\right) .
$$

The case $\beta=0$ is essentially due to Hardy and Littlewood [4] and is also known for $\beta \geqq 0$ (Cheng [2]). For the general case we proceed as follows.

Proof of Lemma 3. As

$$
\begin{aligned}
& S_{\nu}(x)-f(x)=\frac{2}{\pi} \int_{0}^{\pi} \phi(t) \frac{\sin \nu t}{t}+O(1), \\
& \begin{aligned}
\left|S_{\nu}(x)-f(x)\right| & =\frac{2 \nu}{\pi} \int_{0}^{1 / n}|\phi(t)| d t+\frac{2}{\pi}\left|\int_{1 / n}^{\pi} \phi(t) \frac{\sin \nu t}{t} d t\right|+O(1) \\
& =O(\log n)^{\beta}+\frac{2}{\pi}\left|\int_{1 / n}^{\pi} \phi(t) \frac{\sin \nu t}{t} d t\right|+O(1), \quad n \geqq \nu,
\end{aligned}
\end{aligned}
$$

and therefore

$$
\begin{aligned}
\sum_{0}^{n} \mid & S_{\nu}(x)-\left.f(x)\right|^{2} \\
& \leqq K \sum_{0}^{n}\left\{\int_{1 / n}^{\pi} \phi(t) \frac{\sin \nu t}{t} d t\right\}^{2}+O\left(n(\log n)^{2 \beta}\right)+O(n) \\
& =K \int_{1 / n}^{\pi} \int_{1 / n}^{\pi} \frac{\phi(t)}{t} \cdot \frac{\phi(u)}{u}\left(\sum_{0}^{n} \sin \nu t \sin \nu u\right) d t d u+O\left(n(\log n)^{2 \beta}\right)+O(n) \\
& =K T_{n}+O\left(n(\log n)^{2 \beta}\right)+O(n), \quad \text { say },
\end{aligned}
$$

where

$$
\begin{aligned}
2 T_{n}= & \int_{1 / n}^{\pi} \int_{1 / n}^{\pi} \frac{\phi(t)}{t} \frac{\phi(u)}{u}\left\{\frac{\sin \left(n+\frac{1}{2}\right)(u-t)}{2 \sin \frac{1}{2}(u-t)}-\frac{\sin \left(n+\frac{1}{2}\right)(u+t)}{2 \sin \frac{1}{2}(u+t)}\right\} d t d u \\
= & \int_{1 / n}^{\pi} \int_{1 / n}^{\pi} \frac{\phi(t)}{t} \frac{\phi(u)}{u} \frac{\sin n(u-t)}{(u-t)} d t d u \\
& -\int_{1 / n}^{\pi} \int_{1 / n}^{\pi} \frac{\phi(t)}{t} \frac{\phi(u)}{u} \frac{\sin n(u+t)}{(u+t)} d t d u \\
& +O\left(\int_{1 / n}^{\pi} \int_{1 / n}^{\pi} \frac{|\phi(t)|}{t} \frac{|\phi(u)|}{u} d t d u\right) \\
= & T_{n}^{\prime}-T_{n}^{\prime \prime}+O\left(T_{n}^{\prime \prime \prime}\right), \quad \text { say. }
\end{aligned}
$$

As

$$
\int_{1 / n}^{\pi} \frac{|\phi(t)|}{t} d t=\left[\frac{\Phi(t)}{t}\right]_{1 / n}^{\pi}+\int_{1 / n}^{\pi} \frac{\Phi(t)}{t^{2}} d t
$$




$$
\begin{gathered}
\quad= \begin{cases}O(\log n)^{\beta+1}+O(\log \log n), \quad \beta \geqq-1, \\
O(1), \quad \beta<-1,\end{cases} \\
T_{n}^{\prime \prime \prime}=\left\{\begin{array}{l}
O\left((\log n)^{2+2 \beta}\right)+O\left((\log \log n)^{2}\right), \quad \beta \geqq-1, \\
O(1), \quad \beta<-1 .
\end{array}\right.
\end{gathered}
$$

Also

$$
\begin{aligned}
\left|T_{n}^{\prime}\right| & \leqq\left|\int_{1 / n}^{\pi} \frac{\phi(t)}{t} \int_{1 / n}^{t} \frac{\phi(u)}{u} \frac{\sin n(t-u)}{(t-u)} d t d u\right| \\
& \quad+\left|\int_{1 / n}^{\pi} \frac{\phi(t)}{t} \int_{t}^{\pi} \frac{\phi(u)}{u} \frac{\sin n(u-t)}{(u-t)} d u d t\right| \\
& =\left|\int_{1 / n}^{\pi} \frac{\phi(t)}{t} \int_{1 / n}^{t} \frac{\phi(u)}{u} \frac{\sin n(t-u)}{(t-u)} d t d u\right| \\
& \quad+\left|\int_{1 / n}^{\pi} \frac{\phi(u)}{u} \int_{1 / n}^{u} \frac{\phi(t)}{t} \frac{\sin n(u-t)}{(u-t)} d u d t\right| \\
& =2\left|\int_{1 / n}^{\pi} \int_{1 / n}^{t} \frac{\phi(t)}{t} \frac{\phi(u)}{u} \frac{\sin n(t-u)}{(t-u)} d u d t\right| \\
& =2 \mid \int_{1 / n}^{\pi} \frac{\phi(t)}{t^{2}} \int_{1 / n}^{t} \frac{\phi(u)}{(t-u)} \sin n(t-u) d u d t \\
& \leqq 4 n \int_{1 / n}^{\pi} \frac{|\phi(t)|}{t^{2}} \int_{1 / n}^{t}|\phi(u)| d u d t \\
& \leqq 4 n \int_{1 / n}^{\pi} \frac{|\phi(t)|}{t^{2}} \Phi(t) d t .
\end{aligned}
$$

Therefore,

$$
\begin{aligned}
\left|T_{n}^{\prime}\right| & =O\left(n \int_{1 / n}^{\pi} \frac{|\phi(t)|}{t}\left(\log \frac{2 \pi}{t}\right)^{\beta} d t\right) \\
& =O\left(n\left\{\left[\left(\log \frac{2 \pi}{t}\right)^{\beta} \frac{\Phi(t)}{t}\right]_{1 / n}^{\pi}-\int_{1 / n}^{\pi} \Phi(t) d\left|\frac{\left(\log \frac{2 \pi}{t}\right)^{\beta}}{t}\right|\right\}\right) \\
& =O(n)+O\left(n(\log n)^{2 \beta}\right)+O\left(n \int_{1 / n}^{\pi} \frac{\left(\log \frac{2 \pi}{t}\right)^{2 \beta}}{t} d t\right) \\
& =O\left(n h_{n}^{2}\right) .
\end{aligned}
$$

Similarly, we have

$$
\left|T_{n}^{\prime \prime}\right|=\left|\int_{1 / n}^{\pi} \int_{1 / n}^{\pi} \frac{\phi(t)}{t} \frac{\phi(u)}{u} \frac{\sin n(t+u)}{(t+u)} d u d t\right|
$$




$$
\begin{aligned}
& \leqq 2\left|\int_{1 / n}^{\pi} \frac{\phi(t)}{t} \int_{1 / n}^{t} \frac{\phi(u)}{u} \frac{\sin n(t+u)}{(t+u)} d u d t\right| \\
& \leqq 4 n \int_{1 / n}^{\pi} \frac{|\phi(t)|}{t^{2}} \Phi(t) d t \\
& =O\left(n h_{n}^{2}\right) .
\end{aligned}
$$

Thus

$$
\sum_{0}^{n}\left|S_{\nu}(x)-f(x)\right|^{2}=O\left(n h_{n}^{2}\right) \Longrightarrow \sum_{0}^{n}\left|S_{\nu}(x)-f(x)\right|=O\left(n h_{n}\right)
$$

\section{Proof of Theorems.}

5.1. Proof of Theorem 1. We have

$$
\begin{aligned}
& A_{\nu}(x)=\frac{2}{\pi} \int_{0}^{\pi} \phi(t) \cos \nu t d t, \\
& S_{n}(x)-f(x)=\frac{2}{\pi} \int_{0}^{\pi} \phi(t) D_{n}(t) d t,
\end{aligned}
$$

and the series $\sum \varepsilon_{n} A_{n}(x)$ is summable $\left|R, \lambda_{n}, 1\right|$, iff

$$
\begin{aligned}
\sum_{1}^{\infty}\left(\frac{1}{\lambda_{n}}-\frac{1}{\lambda_{n+1}}\right)\left|\sum_{0}^{n} \lambda_{\nu} \varepsilon_{\nu} A_{\nu}(x)\right| & =\frac{2}{\pi} \sum_{1}^{\infty}\left(\frac{1}{\lambda_{n}}-\frac{1}{\lambda_{n+1}}\right)\left|\int_{0}^{\pi} \phi(t) \sum_{0}^{n} \varepsilon_{\nu} \lambda_{\nu} \cos \nu t d t\right| \\
& =\frac{2}{\pi} \sum_{1}^{\infty}\left(\frac{1}{\lambda_{n}}-\frac{1}{\lambda_{n+1}}\right)\left|\int_{0}^{\pi} \phi(t) F_{n}(t) d t\right|<\infty .
\end{aligned}
$$

Therefore, if

$$
S \equiv \sum_{1}^{\infty}\left(\frac{1}{\lambda_{n}}-\frac{1}{\lambda_{n+1}}\right)\left|\int_{0}^{\pi} \phi(t)\left\{F_{n}(t)-\varepsilon_{n} \lambda_{n} D_{n}(t)\right\} d t\right|<K
$$

then

$$
\sum \frac{\mu_{n+1}}{\lambda_{n+1}}\left|\varepsilon_{n}\left\{S_{n}(x)-f(x)\right\}\right|<\infty
$$

is a necessary and sufficient condition for $\sum \varepsilon_{n} A_{n}(x)$ to be summable $\left|R, \lambda_{n}, 1\right|$.

We now proceed to prove $(*)$.

$$
\begin{aligned}
S \leqq \sum_{1}^{\infty}\left(\frac{1}{\lambda_{n}}-\frac{1}{\lambda_{n+1}}\right)\left\{\int_{0}^{1 / n}\left|\phi(t) F_{n}(t)\right| d t+\int_{0}^{1 / n}\left|\varepsilon_{n} \lambda_{n} D_{n}(t) \phi(t)\right| d t\right. \\
\left.+\left|\int_{1 / n}^{\pi} \phi(t)\left(F_{n}(t)-\varepsilon_{n} \lambda_{n} D_{n}(t)\right) d t\right|\right\} \\
=I_{1}+I_{2}+I_{3}, \quad \text { say. }
\end{aligned}
$$

After Lemma 1,

$$
I_{1} \leqq K_{1} \sum_{1}^{\infty}\left(\frac{1}{\lambda_{n}}-\frac{1}{\lambda_{n+1}}\right)\left|\varepsilon_{n}\right| \int_{0}^{1 / n}\left|\phi(t) E_{n}(t)\right| d t
$$




$$
\begin{aligned}
& +K_{2} \sum_{1}^{\infty}\left(\frac{1}{\lambda_{n}}-\frac{1}{\lambda_{n+1}}\right)\left(\sum_{0}^{n-1}\left|\left(\Delta \varepsilon_{\nu}\right) \int_{0}^{1 / n} \phi(t) E_{\nu}(t) d t\right|\right) \\
\leqq & K_{1} \sum_{1}^{\infty} \frac{\mu_{n+1}}{\lambda_{n+1}} n\left|\varepsilon_{n}\right| \Phi(1 / n)+K_{2} \sum_{\nu=0}^{\infty} \nu \lambda_{\nu}\left|\Delta \varepsilon_{\nu}\right| \sum_{n=\nu+1}^{\infty} \Phi(1 / n)\left(\frac{1}{\lambda_{n}}-\frac{1}{\lambda_{n+1}}\right) \\
\leqq & K_{3}+K_{4} \sum_{\nu=0}^{\infty} \lambda_{\nu}\left|\Delta \varepsilon_{\nu}\right| \sum_{\nu+1}^{\infty} \psi_{n} \Delta\left(1 / \lambda_{n}\right) \\
< & K \\
I_{2} \leqq & K_{1} \sum_{0}^{\infty} \frac{\mu_{n+1}}{\lambda_{n+1}}\left|\varepsilon_{n}\right| \psi_{n}<K
\end{aligned}
$$

and

$$
\begin{aligned}
I_{3} \leqq & K_{1} \sum_{n=1}^{\infty}\left(\frac{1}{\lambda_{n}}-\frac{1}{\lambda_{n+1}}\right) \sum_{\nu=0}^{n-1}\left|\Delta \varepsilon_{\nu}\right| \int_{1 / n}^{\pi}\left|E_{\nu}(t) \phi(t)\right| d t \\
& +K_{2} \sum_{n=1}^{\infty}\left(\frac{1}{\lambda_{n}}-\frac{1}{\lambda_{n+1}}\right)\left|\varepsilon_{n} \sum_{\nu=1}^{n} \mu_{\nu} \int_{1 / n}^{\pi} \phi(t) D_{\nu-1}(t) d t\right| \\
\leqq & K_{1} \sum_{\nu=0}^{\infty}\left|\Delta \varepsilon_{\nu}\right| \lambda_{\nu} \sum_{n=\nu+1}^{\infty}\left(\frac{1}{\lambda_{n}}-\frac{1}{\lambda_{n+1}}\right) \int_{1 / n}^{\pi} \frac{|\phi(t)|}{t} d t+K_{2} \\
\leqq & K_{1} \sum_{0}^{\infty}\left|\Delta \varepsilon_{\nu}\right| \lambda_{\nu} \sum_{\nu+1}^{\infty} p_{n} \Delta\left(1 / \lambda_{n}\right)+K_{2} \\
& <K .
\end{aligned}
$$

This completes the proof of the theorem.

5.2. Proof of Theorem 2. After Theorem 1, it is enough to note that when $\psi(1 / t)=\left(\log \frac{2 \pi}{t}\right)^{\beta}$, and $\lambda_{n}=n$,

$$
\begin{aligned}
& p_{n}=\int_{1 / n}^{\pi} \frac{|\phi(t)|}{t} d t=O\left(k_{n}\right) ; \\
& \sum_{n=\nu+1}^{\infty} p_{n} \Delta\left(\frac{1}{\lambda_{n}}\right)=O\left(\frac{k_{\nu}}{\nu}\right) ;
\end{aligned}
$$

for $t \in\left[\frac{1}{n}, \pi\right]$,

$$
\left|\sum_{1}^{n} \mu_{\nu} D_{\nu-1}(t)\right|=O\left(\frac{1}{t^{2}}\right)
$$

and therefore

$$
\begin{aligned}
\left|\sum_{1}^{n} \mu_{\nu} \int_{1 / n}^{\pi} \phi(t) D_{\nu-1}(t) d t\right| & \leqq K_{1} \int_{1 / n}^{\pi} \frac{|\phi(t)|}{t^{2}} d t \\
& =K_{1}\left|\frac{\Phi(t)}{t^{2}}\right|_{1 / n}^{\pi}+2 K_{2} \int_{1 / n}^{\pi} \frac{\Phi(t)}{t^{3}} d t \\
& =K n(\log n)^{\beta} .
\end{aligned}
$$


5.3. Proof of Theorem 3. In view of Lemma 2 we may employ summability method $\left|R,(\log n)^{r}, 1\right|, r>0$, instead of method $|R, \log n, 1|$. For the sake of convenience in further analysis we choose $r>1+|\beta|$. As in the case of Theorem 2 , we again deduce this theorem from Theorem 1 . Here

$$
\begin{aligned}
\mu_{n} & =(\log (n+1))^{r}-(\log n)^{r} \\
& =r \frac{(\log n)^{r-1}}{n}+O\left(\frac{(\log n)^{r-1}}{n^{2}}\right),
\end{aligned}
$$

and therefore for $t \in\left[\frac{1}{n}, \pi\right]$

$$
\begin{aligned}
& \left|\sum_{1}^{n} \mu_{\nu} \sin \left(\nu-\frac{1}{2}\right) t\right| \\
& \quad \leqq r\left|\sum_{1}^{n} \frac{(\log \nu)^{r-1} \sin \left(\nu-\frac{1}{2}\right) t}{\nu}\right|+K \sum_{1}^{n}(\log \nu)^{r-1} \frac{\left|\sin \left(\nu-\frac{1}{2}\right) t\right|}{\nu^{2}} \\
& \quad \leqq K+r\left|\sum_{\nu \leqq 2 \pi / t}(\log \nu)^{r-1} \frac{\sin \left(\nu-\frac{1}{2}\right) t}{\nu}\right|+r\left|\sum_{2 \pi / t<\nu} \frac{(\log \nu)^{r-1}}{\nu} \sin \left(\nu-\frac{1}{2}\right) t\right| \\
& \quad \leqq K_{1}\left(\log \frac{2 \pi}{t}\right)^{r-1}+K_{2} \frac{(\log (2 \pi / t))^{r-1}}{(2 \pi / t)}(2 \pi / t) \\
& \quad \leqq K\left(\log \frac{2 \pi}{t}\right)^{r-1} .
\end{aligned}
$$

Hence

$$
\begin{aligned}
& \left|\sum_{1}^{n} \mu_{\nu} \int_{1 / n}^{\pi} \phi(t) D_{\nu-1}(t) d t\right| \\
& \quad \leqq K \int_{1 / n}^{\pi} \frac{|\phi(t)|(\log (2 \pi / t))^{r-1}}{t} d t \\
& \quad \leqq K\left[\left(\log \frac{2 \pi}{t}\right)^{r-1} \frac{\Phi(t)}{t}\right]_{1 / n}^{\pi}+K_{1} \int_{1 / n}^{\pi} \Phi(t)\left|d\left(\frac{\log (2 \pi / t))^{r-1}}{t}\right)\right| \\
& \quad \leqq K_{2}(\log n)^{\beta+r}
\end{aligned}
$$

which implies

$$
\begin{aligned}
\sum_{1}^{\infty} \Delta\left(\frac{1}{\lambda_{n}}\right)\left|\varepsilon_{n} \sum_{1}^{n} \mu_{\nu} \int_{1 / n}^{\pi} \phi(t) D_{\nu-1}(t) d t\right| & \leqq K \sum \frac{\left|\varepsilon_{n}\right|(\log n)^{\beta+r}}{n(\log n)^{r+1}} \\
& \leqq K
\end{aligned}
$$

Again, as $p_{n}=O\left(k_{n}\right)$, the proof of Theorem 3 is completed.

\subsection{Corollary to Theorem 3 .}

The following corollary is an interesting special case of Theorem 3.

Corollary. If $\Phi(t)=O\left(t\left(\log \frac{2 \pi}{t}\right)^{-\eta}\right), \eta>0$, then $\Sigma A_{n}(x)$ is summable 
$|R, \log n, 1|$, iff

$$
\Sigma \frac{1}{n \log n}\left|S_{n}(x)-f(x)\right|<\infty .
$$

Proof of Tneorem 4. Let $\left\{\gamma_{n}\right\}$ be a given sequence. After Lemma 3 we have

$$
\begin{aligned}
\sum_{0}^{n} \gamma_{\nu}\left|S_{\nu}(x)-f(x)\right| & =\sum_{0}^{n-1} \Delta\left(\gamma_{\nu}\right) \Sigma\left|S_{k}(x)-f(x)\right|+\gamma_{n} \sum_{0}^{n}\left|S_{\nu}(x)-f(x)\right| \\
& =O\left(\sum_{0}^{n-1} \nu h_{n}\left|\Delta \gamma_{\nu}\right|\right)+O\left(n h_{n} \gamma_{n}\right) .
\end{aligned}
$$

Case $I \cdot$ Take $\left\{\gamma_{n}\right\}=\left\{\frac{\varepsilon_{n}}{n}\right\}$ so that $n \gamma_{n}=\varepsilon_{n}$ and $(n+1) \Delta \gamma_{n}=\frac{\varepsilon_{n}}{n}+\Delta \varepsilon_{n}$, and the result follows from the sufficiency part of Theorem 2 .

Case II: Take $\left\{\gamma_{n}\right\}=\left\{\frac{\varepsilon_{n}}{n \log n}\right\}$. Here we have

$$
(n+1) \Delta \gamma_{n}=\left(\frac{\Delta \varepsilon_{n}}{\log (n+1)}\right)+O\left(\frac{\left|\varepsilon_{n}\right|}{n \log n}\right) \text {. }
$$

Now we get the result in the light of Theorem 3 .

\section{REFERENCES}

[1] Chandrasekharan, K. and Minakshisundaram, S., Typical means. Oxford University Press, 1952.

[2] Cheng, M.-T., Summability factors of Fourier series at a given point. Duke Mathematical Journ., 14 (1947), 405-410.

[3] Dikshit, G.D., Localization relating to the summability $\left|R, \lambda_{n}, 1\right|$ of Fourier series. Indian Journ. Math., 7 (1965), 31-39.

[4] Hardy, G.H. AND Littlewood, J.E., The strong summability of Fourier series. Fundamenta Mathematicae XXV (1935), 162-189.

[5] Hsiang, F.C., On the absolute summability factors of Fourier series at a given point. Composito Mathematico, 17 (1966), 156-160.

[6] LiU, T.-S., On the absolute Cesàro summability factors of Fourier seaies. Proc. Japan Acad., 41 (1965), 757-762.

[7] Matsumoto, K., Local property of the summability $\left|R, \lambda_{n}, 1\right|$. Tôhoku Math. Journ. (2) 8 (1956), 114-124.

[8] Pati, T., On an unsolved problem in the theory of absolute summability factors of Fourier series. Math. Zeits., 82 (1963), 106-114.

[9] Prasad, B. N. AND Bhatt, S. N., The summability factors of a Fourier series. Duke Math. Journ., 24 (1957), 103-117.

[10] WANG, S.-L., A boundary problem in theory of absolute summability factors of Fourier series. Acta Math. Sinica, 16 (1966), 503-512 (Chinese) : translated as Chinese Math.-Acta, 8 (1966), 524-533.

Department of Mathematics

UNIVERSITY OF AUCKLAND

New Zealand 